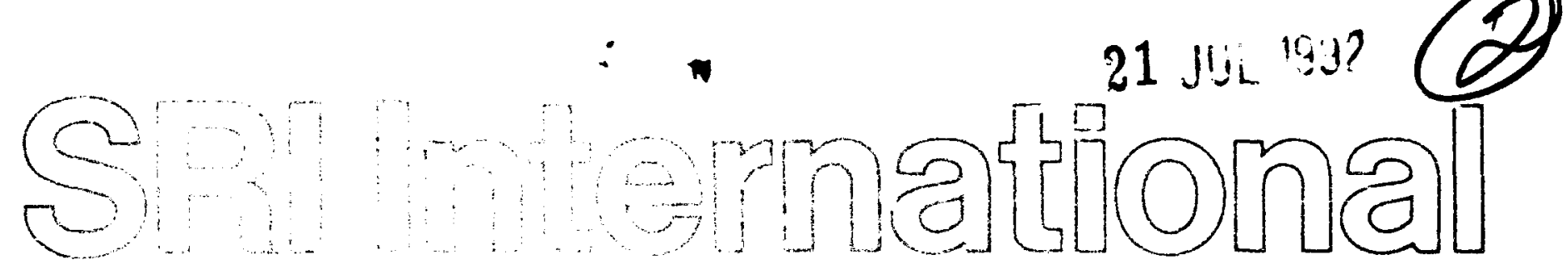

AD-A254 709

AFOSR.TR. $\cong 2 \quad 065$

\title{
Novel Nonlinear Laser Diagnostic Techniques
}

Prepared by:

Gregory W. Faris,Jay B. Jeffries, and David L. Huestis

Molecular Physics Laboratory

Contract No. F49620-90-C-0044

SRI Project PYU 1187

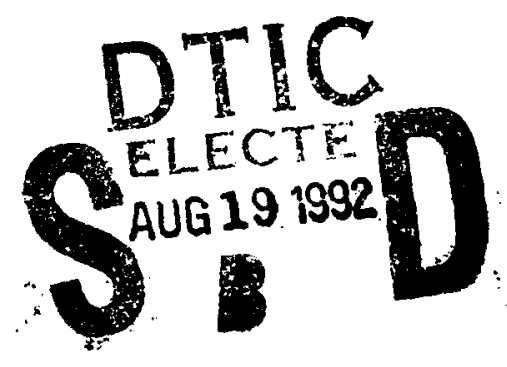

MP 92-142

Prepared for:

Air Force Office Of Scientific Research

Building 410

Bolling AFB, DC $20332-6448$

Attn: Dr. Julian Tishkoff

Approved:

Donald J. Eckstrom, Director

Molecular Physics Laboratory

David M. Golden

Vice President

Physical Sciences Division

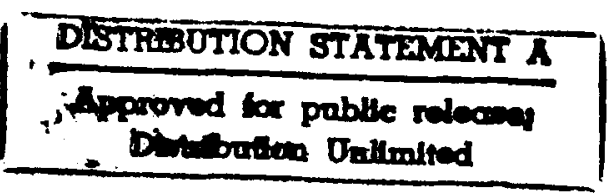

\section{$\begin{array}{llll}92 & 8 & 18 & 0.98\end{array}$}

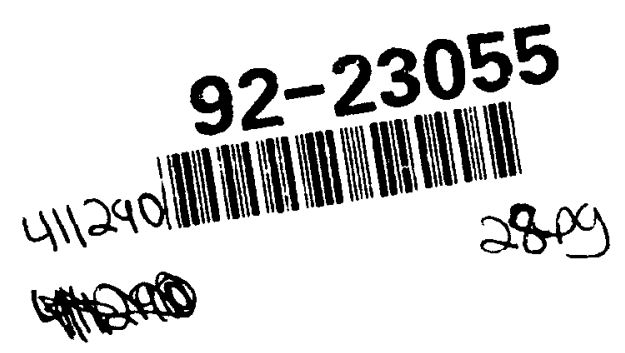




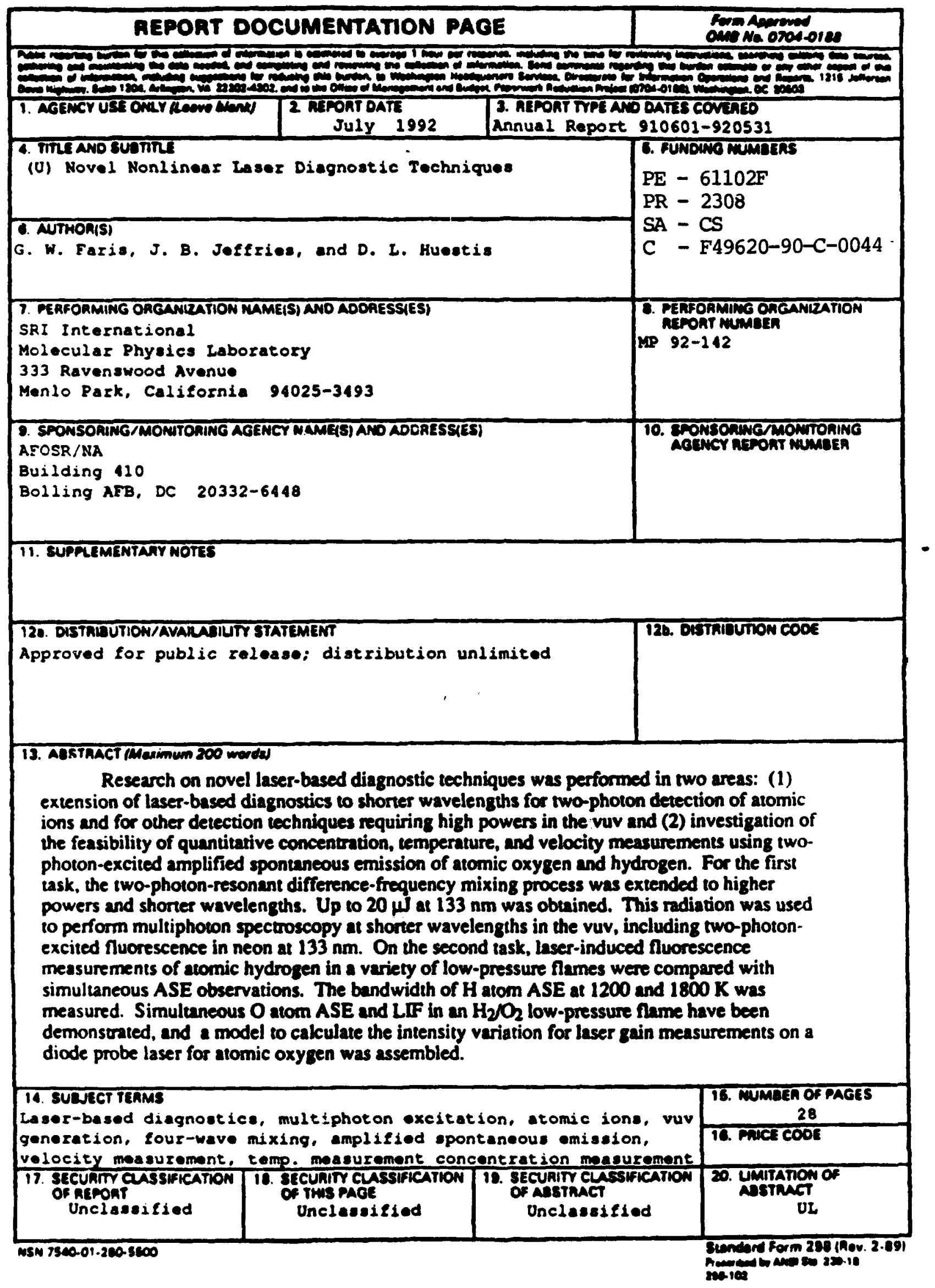




\section{CONTENTS}

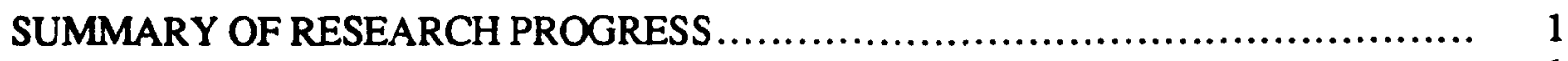

Objectives....................................................................... 1

Task 1: VUV Laser-Based Diagnostics .......................................... 1

Task 2: Amplified Spontaneous Emission......................................... 15

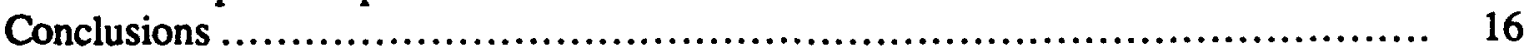

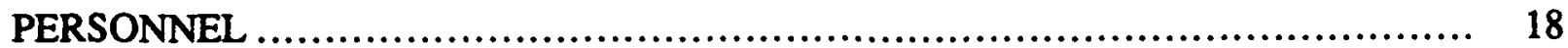

PUBLICATIONS ....................................................................... 19

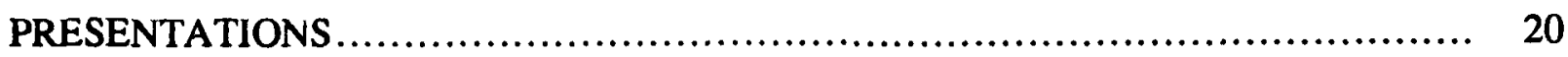

PROJECT INTERACTIONS ......................................................... 21

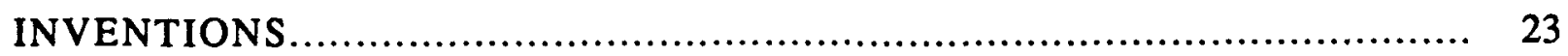

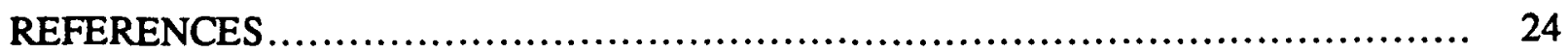

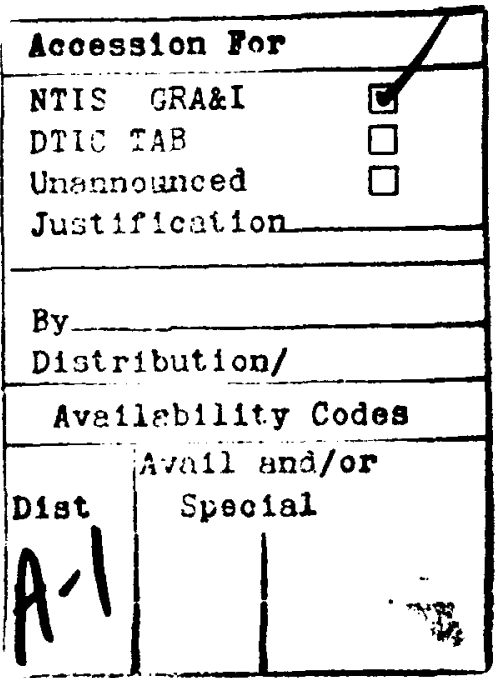




\section{SUMMARY OF RESEARCH PROGRESS}

\section{OBJECTIVES}

The research for Task 1 is centered on extending laser-based diagnostics to shorter wavelengths. The objectives of this task are to develop new vuv laser sources and techniques, with emphasis on widely tunable radiation with high powers, and to apply this vuv radiation to diagnostics of species not accessible with current visible and near ultraviolet laser sources.

Task 2 investigates the feasibility of quantitative concentration, gas temperature, and velocity measurement of atomic hydrogen and oxygen using laser-excited amplified spontaneous emission (ASE).

\section{TASK 1: VUV LASER-BASED DIAGNOSTICS}

\section{Approach and Progress in Past Year}

Advances in laser techniques and technology have been a strong driving force in the development of nonintrusive diagnostics techniques. The production and use of higher laser powers at shorter wavelengths have been particularly fruitful throughout the 1980 s and have led to the now well-established techniques of planar laser-induced fluorescence, two-photon-excited fluorescence, and resonantly enhanced multiphoton ionization. More recent advances in nonlinear crystals, such as $\beta-\mathrm{BaB}_{2} \mathrm{O}_{4}(\mathrm{BBO})$ and $\mathrm{LiB}_{3} \mathrm{O}_{5}(\mathrm{LBO})$, allow use of these techniques on species requiring shorter wavelengths.

Below $200 \mathrm{~nm}$, the generation and application of laser radiation become more difficult for two reasons: the lack of suitable nonlinear frequency-converting crystals and the increasing absorption of background gases in this region. However, a number of diagnostic problems can be solved only in the vuv. Some species, such as light atomic ions, can be excited only by two photons of vuv radiation (three-photon-resonant excitation is possible in principle, but the process is very weak and the required laser intensities are correspondingly very large).

For light atoms and molecules, single photon vuv excitation offers several advantages over two-photon excitation. Because high intensities are not required, photodissociation or other perturbing processes can be avoided. Quantitative results are simpler to obtain because the process is linear and the transition strengths are often well known. The single photon sensitivity is higher, 
and planar imaging may be possible. Through $1+1$ multiphoton ionization (MPI) coupled with mass spectrometry, ultrasensitive measurements can be made, comparable to those achievable with a gas chromatograph-mass spectroscopy system, except with a much faster response time. VUV diagnostics will be useful for plasma diagnostics, the study of chemical dynamics and kinetics of processes important in combustion and fluid flow, the calibration of other diagnostic techniques, and the study of shock-heated flows.

A key part of this research is the production of high power vuv radiation suitable for diagnostics measurements. Because of the lack of suitable nonlinear crystals for vuv generation, frequency conversion must be performed in gases. Because gases are centrosymmetric, fourwave-mixing is the lowest order frequency conversion process that may be used. To obtain high powers, techniques using resonances are required, such as multi-order anti-Stokes Raman shifting and two-photon-resonant sum- and difference-frequency mixing.

Under a previous contract, Faris and coworkers (1990) investigated multi-order Raman shifting for the two-photon excitation of atomic fluorine and molecular fluorine. Wavelengths as short as $170 \mathrm{~nm}$ were produced. However, the Raman shifting has limitations for vuv generation, including low efficiency for shorter vuv wavelengths, large intensity fluctuations when shifting dye lasers, and limited tuning range when shifting excimer lasers. Raman shifting can be the most appropriate source for certain applications, for example, when the wavelengths required lie within the tuning range of one of the Raman orders for shifting the ArF laser. Examples of such fortuitous overlaps are the use of a Raman-shifted ArF laser for two-photon excited detection of atomic and molecular fluorine using the second anti-Stokes line from $\mathrm{HD}$ and the first Stokes line from $D_{2}$, respectively.

The two-photon-resonant sum- and difference-frequency process is shown in Figure 1. Because of the two-photon resonance, this mixing process can provide efficiencies of about $10^{-4}$, significantly better than frequency tripling, but it requres two lasers. For vuv generation, the difference frequency process is preferable because much of the vuv spectral region can be covered, and negative dispersion of the medium is not required. The difference-frequency process has been demonstrated using the $5 \mathrm{p}[5 / 2,2] \leftarrow 4 \mathrm{p}^{6}{ }^{1} \mathrm{~S}_{\mathrm{o}}$ (Hilber et al., 1987) and $5 \mathrm{p}[1 / 2,0] \leftarrow 4 \mathrm{p}^{6}{ }^{1} \mathrm{~S}_{\mathrm{o}}$ (Marangos et al., 1990) resonances in krypton, using frequency-doubled dye laser radiation as the two-photon pump laser. Because the vuv output power scales as the square of the two-photon pump laser intensity and minimal tuning of the pump laser is required, frequency-doubled dye lasers may not be the best pump laser. 


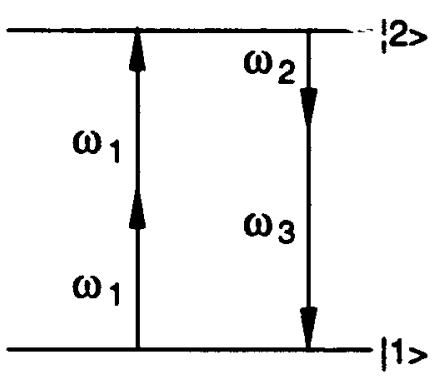

(a)

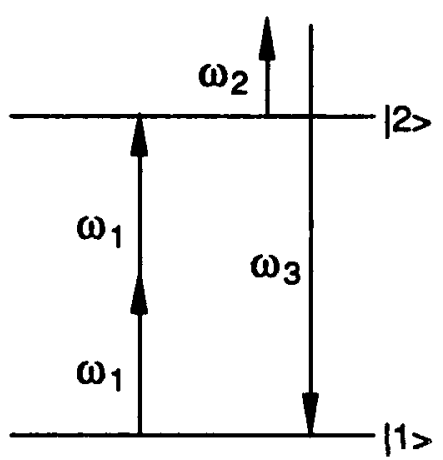

(b)

CM-1187-20

Figure 1. Two-photon-resonant difference-(a) and sum-(b) frequency mixing. 
By coincidence, there are two-photon resonances in krypton, $\mathrm{H}_{2}$, and $\mathrm{HD}$ within the tuning range of the ArF laser. Because of the very high powers attainable from the ArF laser, this is an attractive pump laser for two-photon-resonant difference-frequency generation. Use of the $6 p[3 / 2,2] \leftarrow 4 p^{6}{ }^{1} S_{o}$ transition in krypton for vuv-xuv generation with an ArF laser was proposed by Hilbig et al.(1986). Two-photon-resonant sum-frequency mixing using the $E, F\left(v^{\prime}=6\right)^{1} \Sigma_{g}^{+}$ $\leftarrow \mathrm{X}^{1} \Sigma_{\mathrm{g}}^{+}\left(\mathrm{v}^{\prime \prime}=0\right) \mathrm{Q}(1)$ transition of $\mathrm{H}_{2}$ with an $\mathrm{ArF}$ laser has been demonstrated for xuv generation (Okada et al., 1990).

Two-photon-resonant difference-frequency generation of vuv using an ArF laser was not demonstrated until last year, when it was reported by Faris and Dyer (1991) and Strauss and Funk (1991). The advantages of this technique over mixing techniques using frequency-doubled dye lasers as the two-photon pump laser include the higher power available from the ArF excimer laser, the ability to tune to shorter wavelengths (tuning from $110 \mathrm{~nm}$ to $180 \mathrm{~nm}$ is possible), and the lower sensitivity to phase mismatch due to the shorter two-photon pump wavelength.

The apparatus we use for two-photon-resonant difference-frequency mixing is shown in Figure 2. The excimer laser is a dual discharge laser (Lambda Physik Model 150). Modifications were made to this laser to improve the laser mode quality for previous work on Raman-shifting. The excimer laser is run as an oscillator-triple pass amplifier. Two mirrors and a pinhole between the oscillator and amplifier are used to filter the beam spatially and to magnify the beam size. The output, up to $60 \mathrm{~mJ}$ in a beam five times over the diffraction limit, is combined with the frequencydoubled output of a dye laser (Quanta-Ray PDL) pumped by a Nd:YAG-pumped laser (QuantaRay DCR II) on a dichroic beamsplitter. The timing of the two laser pulses is synchronized to about $1 \mathrm{~ns}$. A feedback circuit corrects for drift in the timing of the excimer laser pulse. The two beams are focused individually with $1-\mathrm{m}$ lenses into a gas cell. The beam path of the ArF laser is purged with argon to minimize the effects of oxygen Schumann Runge absorption. The beam path from the beam splitter on is evacuated. Light passing through the gas cell is collimated with a $\mathrm{MgF}_{2}$ lens and continues into a vacuum spectrometer or is dispersed with a $\mathrm{MgF}_{2}$ Pellin Broca prism.

During the past year, we have improved the performance of the mixing system, leading to higher powers and shorter wavelengths, and have applied the vuv radiation to multiphoton detection of atoms. In our previous work on producing wavelengths near $147 \mathrm{~nm}$, we were limited to mixing in krypton because of the strong amplified spontaneous emission when exciting the $E, F\left(v^{\prime}=6\right){ }^{1} \Sigma_{g}^{+} \leftarrow X{ }^{1} \Sigma_{g}^{+}\left(v^{\prime \prime}=0\right) Q(1)$ transition in $\mathrm{H}_{2}$ (Pummer et al,. 1983), which made monitoring the mixing radiation difficult. This radiation is strongest 


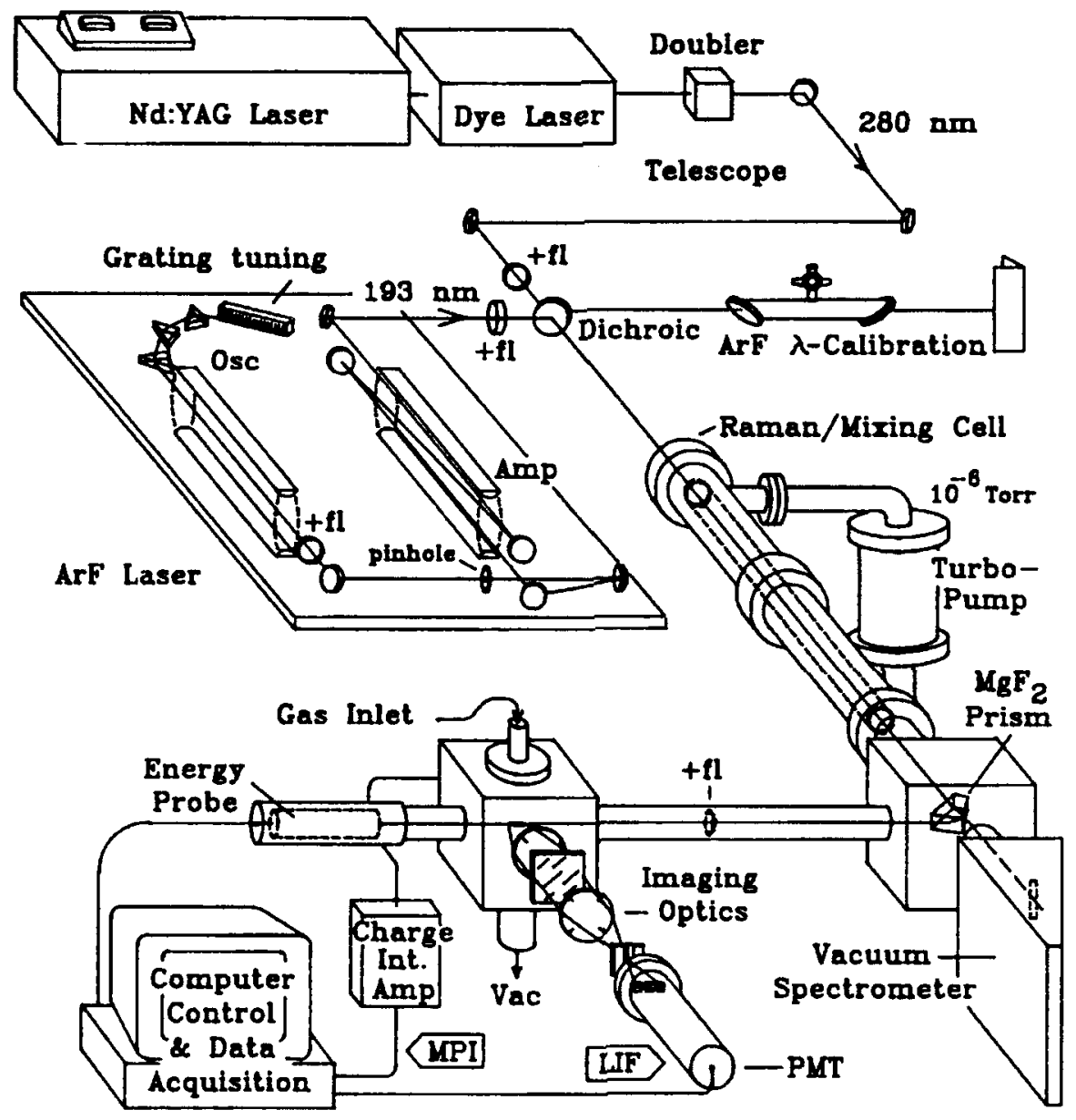

CM-1187-34

Figure 2. Experimental arrangement for two-photon-resonant difference-frequency generation. 
between about 140 and $160 \mathrm{~nm}$. Mixing in krypton at shorter wavelengths leads to lower efficiencies than at longer wavelengths. This is illustrated in Figure 3, which shows the pressure dependence for production of $133-\mathrm{nm}$ radiation in krypton. The peak energy is only $2 \mu \mathrm{J}$, significantly less than that obtained near $147 \mathrm{~nm}$ in krypton $(6 \mu \mathrm{J})$. We have performed mixing in $\mathrm{H}_{2}$ to produce $133 \mathrm{~nm}$ and found that the ASE is weak enough in this region to allow use of the mixing process. Figure 4 shows pressure dependencies of the ASE at $145 \mathrm{~nm}$ and mixing to produce $134 \mathrm{~nm}$. By operating at a lower pressure of $\mathrm{H}_{2}$, we can further reduce the ASE. We have obtained up to $20 \mu \mathrm{J}$ at $134 \mathrm{~nm}$ by mixing in $\mathrm{H}_{2}$. This energy is adequate for nonlinear optical diagnostic techniques such as two-photon-excited fluorescence.

We have applied the vuv radiation to two experiments to examine feasibility of multiphoton techniques at short vuv wavelengths. By performing multiphoton excitation on noble gases, we can examine basic questions concerning sensitivity and technological complications without the additional experimental difficulty of the production of atomic ions. We have investigated two systems: $1+1$ resonantly enhanced multiphoton ionization (REMPI) of atomic xenon and twophoton excited fluorescence of atomic neon.

We used two photons at $147 \mathrm{~nm}$ to perform $1+1$ REMPI through the $5 \mathrm{p}^{5} 6 \mathrm{~s}[3 / 2,1]$ state of xenon as shown in Figure 5. The 147-nm radiation was produced through mixing in krypton. Because vuv radiation can readily ionize many molecular species, background ion signals are a major consideration for vuv REMPI. With energies of only $3 \mu \mathrm{J}$, we have obtained signal-to-noise ratios of $>50$ for the ion signal, indicating that this approach can give useful signals. A $1+1$ MPI and absorption spectrum for $\mathrm{Xe}$ are shown in Figure 6. The strong resonance absorption in xenon leads to a dip in the MPI signal at line center. A power dependence of the ion signal from xenon is shown in Figure 7. From the figure, it is apparent that the signal follows the expected square dependence on the vuv power.

Because the signal relies on detection of ions, $1+1$ REMPI is not very well suited to detection in plasmas. However, $1+1$ REMPI might be performed in plasmas in conjunction with optogalvanic detection. In addition, 1+1 REMPI with vuv radiation is useful when high sensitivity measurements are required, such as for resonant ionization mass spectroscopy of trace species, when two-photon techniques lead to photodissociation or other production or destruction mechanisms that can cause detection errors, and as a calibration procedure for two-photon excitation. 


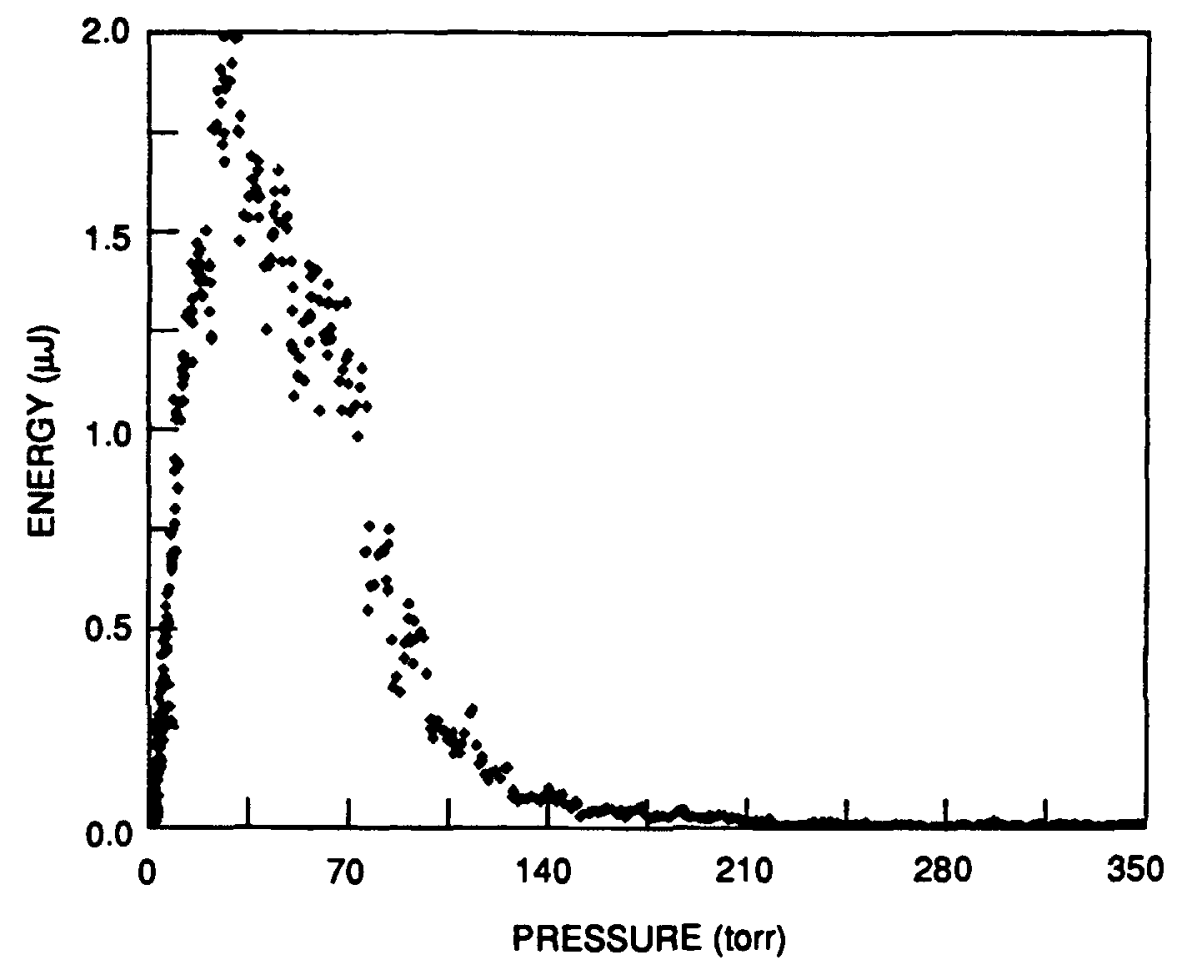

CM-1187-35

Figure 3. Pressure dependence of vacuum ultraviolet radiation for mixing in krypton. 


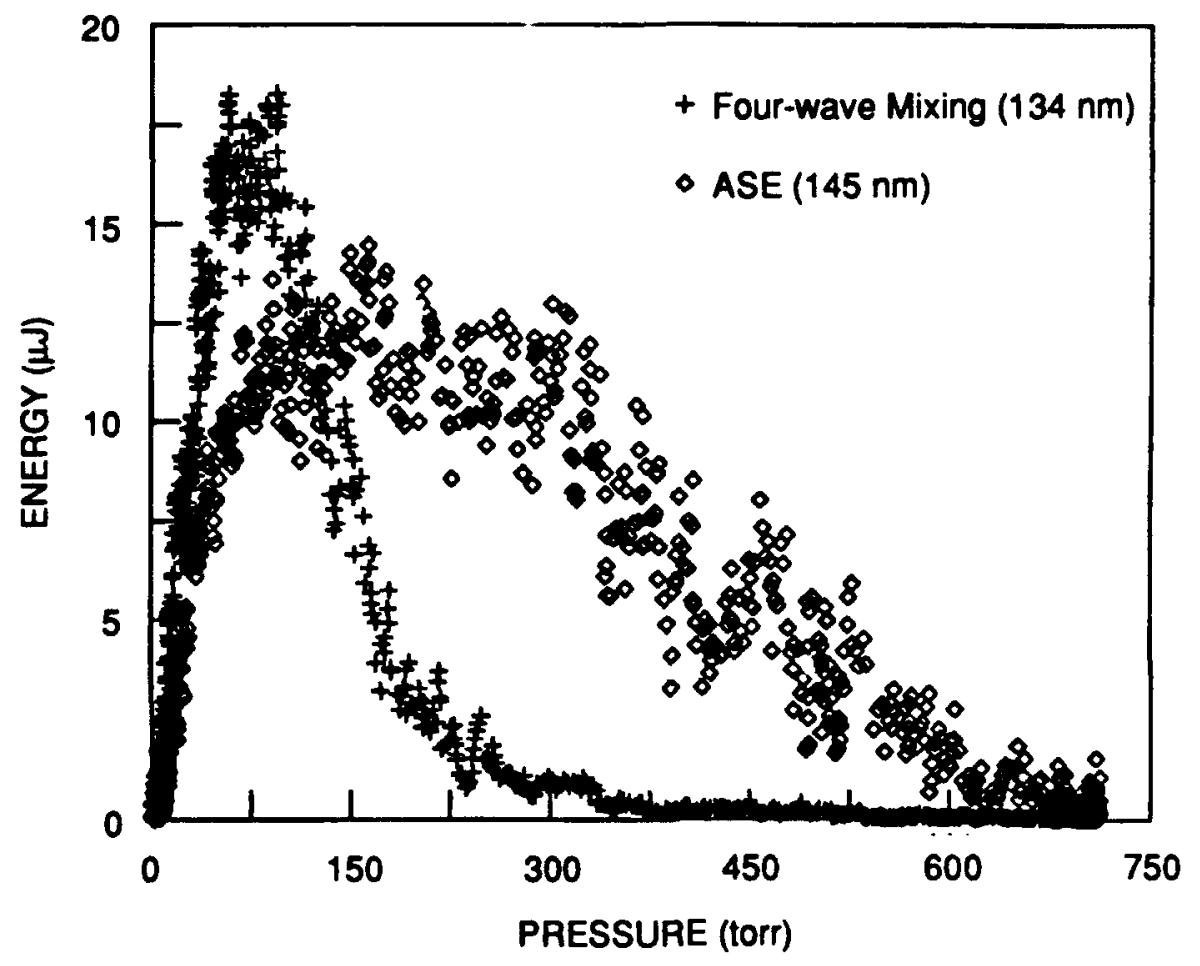

CM-1187-36

Figure 4. Pressure dependence of vacuum ultraviolet radiation for mixing and $A S E$ in $\mathrm{H}_{2}$. 
For demonstration of two-photon excited fluorescence at shorter wavelengths in the vav, we have chosen neon, which, with the first two-photon resonance corresponding to $\sim 133 \mathrm{~nm}$, is the second most difficult neutral atom to excite after helium. We have been able to observe fluorescence following two-photon resonant excitation of the $2 p^{5} 3 p[3 / 2,2]$ state of neon from the ground state using the excitation scheme shown in Figure 8. Fluorescence is detected using a photomultiplier and a 700-nm short pass filter. An excitation spectrum for this transition is shown in Figure 9 for a pressure of 100 torr of neon. The signal is currently fairly weak, on the level a few photons per shot, but the system has not been optimized. These initial measurements on twophoton-excited fluorescence in neon indicate the feasibility of two-photon-excited fluorescence in atcmic ions through excitation with radiation produced by two-photon-resonant differencefrequency mixing. This is but one of many interesting diagnostic techniques possible with high power vuv radiation. 


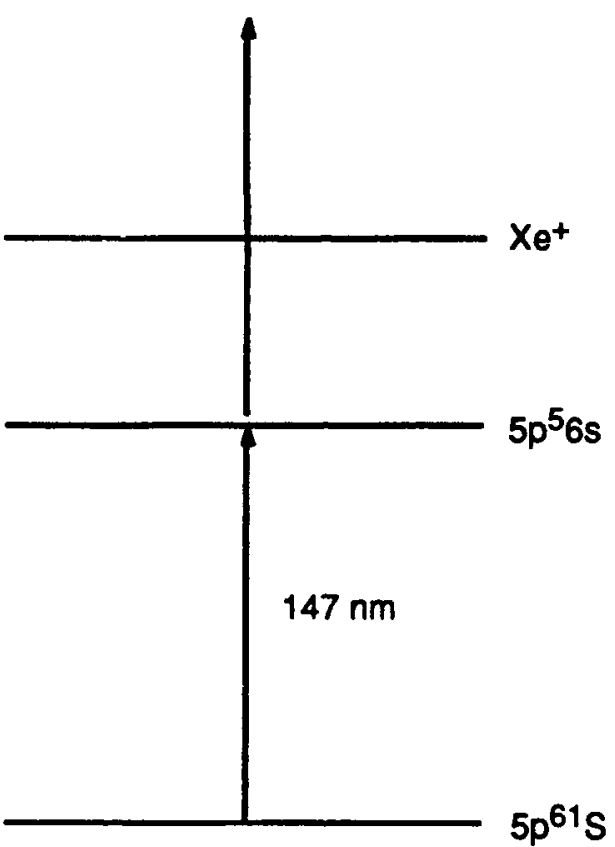

CM-1187-37

Figure 5. Energy level diagram for $1+1$ MPI in xenon at $147 \mathrm{~nm}$. 


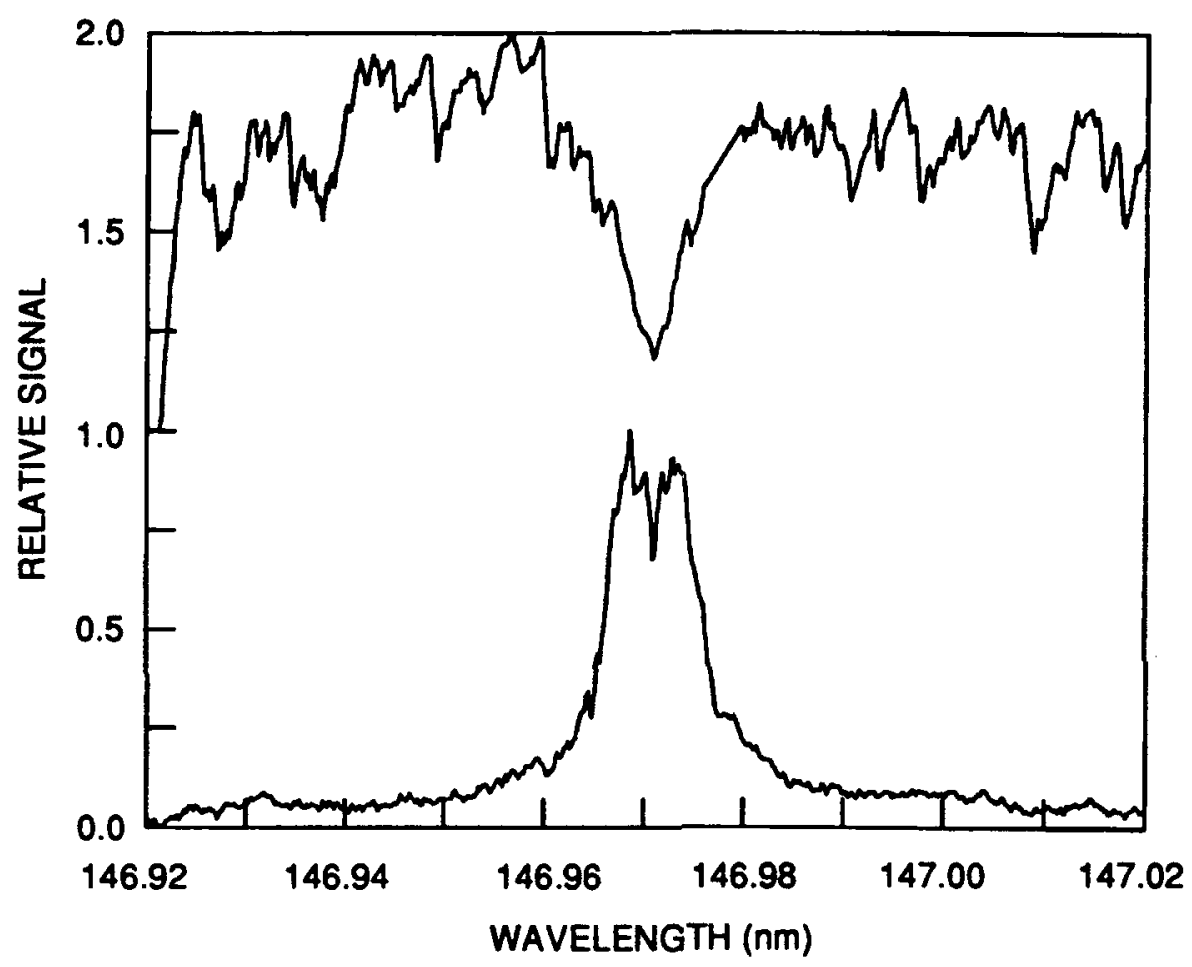

CM-1187-38

Figure 6. Absorption and $1+1 \mathrm{MPI}$ spectra for xenon with $147-\mathrm{nm}$ radiation. 


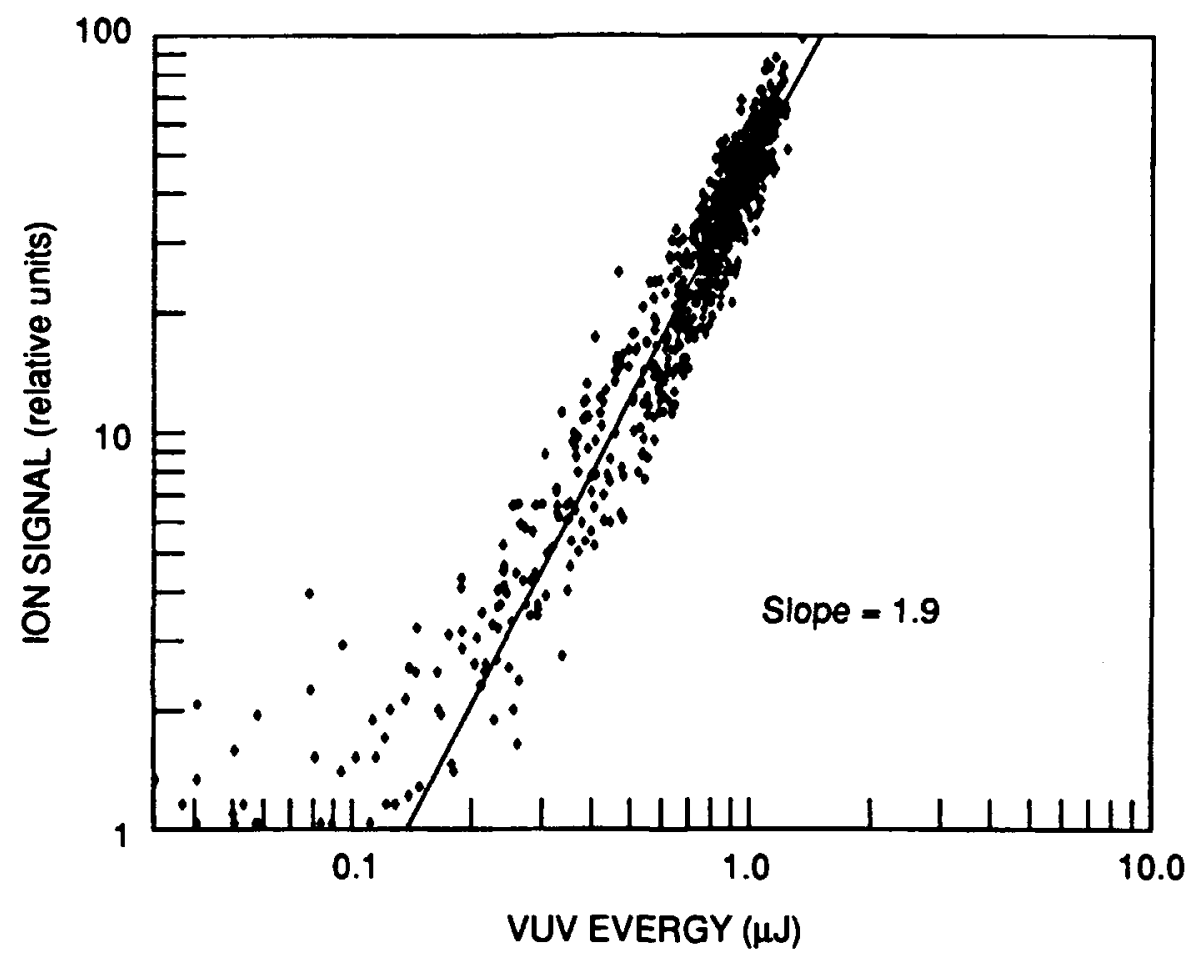

CM-1187-39

Figure 7. Power dependence of ion signal from 1+1 REMPI in xenon. 


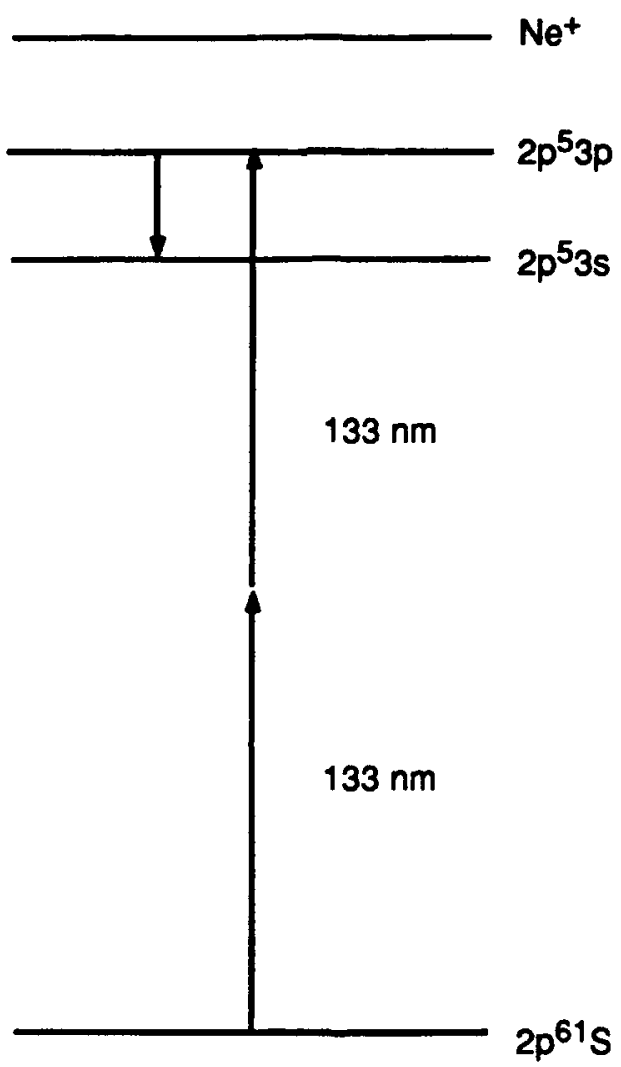

CM-1187-40

Figure 8. Energy level diagram for twophoton-excited fluorescence in neon at $147 \mathrm{~nm}$. 


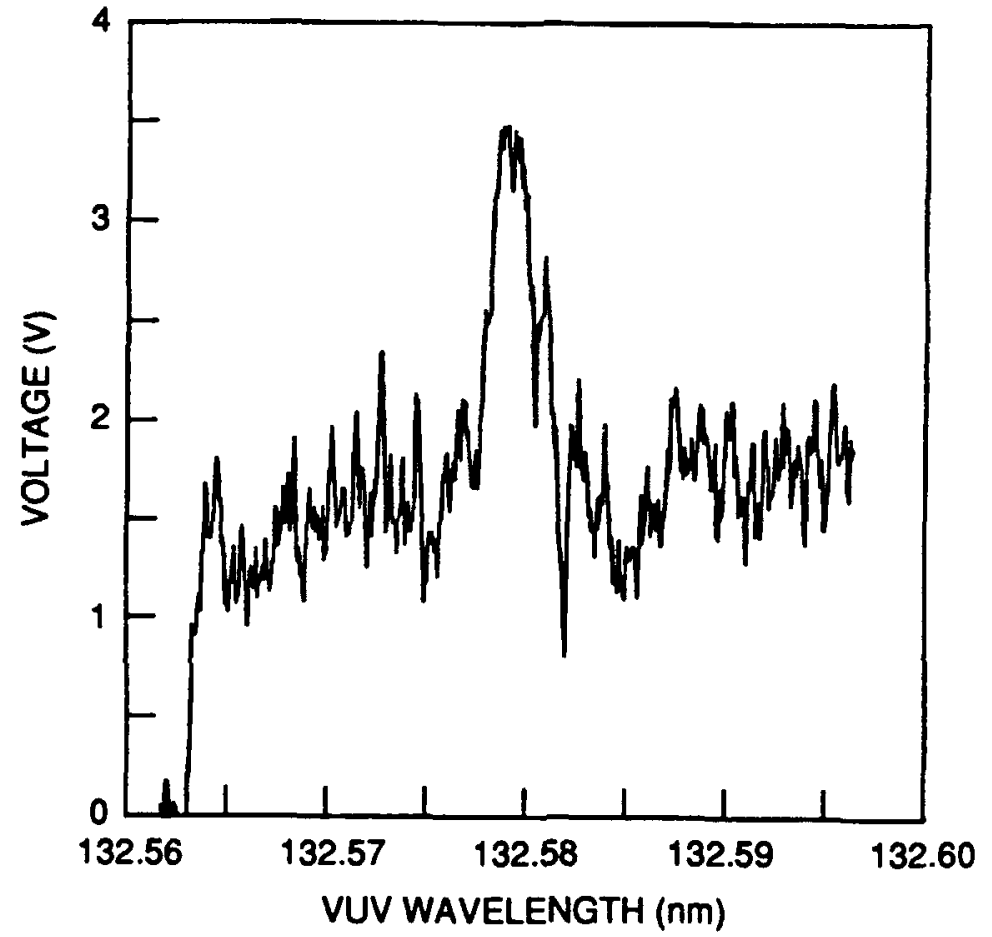

CM-1187-41

Figure 9. Two-photon-excited fluorescence signal in neon with 133-nm radiation. 


\section{TASK 2: AMPLIFIED SPONTANEOUS EMISSION}

Atoms can be excited by multiphoton laser excitation in flames, plasmas, and reacting flows and subsequently detected by fluorescence and ionization techniques. Two-photon selection

rules produce atoms in excited states that do not have allowed one-photon transitions to the ground state but instead radiate to an intermediate level. For light atoms like hydrogen and oxygen, important in supersonic aircraft engines, these intermediate states are more than $10 \mathrm{eV}$ above the ground state. Thus, at combustion temperatures, there is little thermal population in these intermediate states. As soon as a significant number of ground state atoms are two-photon excited, there is a population inversion between the excited and intermediate states and spontaneous emission along the excitation direction can experience gain. Such gain produces amplified spontaneous emission (ASE) directed forward and backward along the laser beam.

During the past year, we have studied two-photon ASE of atomic hydrogen and oxygen in a variety of flames. Two photons near $205 \mathrm{~nm}$ excited the $3 \mathrm{~s}$ and $3 \mathrm{~d}$ states of hydrogen, which subsequently radiate Balmer $\alpha$ near $656 \mathrm{~nm}$; two photons near $226 \mathrm{~nm}$ excite oxygen to the $3 \mathrm{p}{ }^{3} \mathrm{P}$ state, which subsequently radiates near $845 \mathrm{~nm}$ to the $3 \mathrm{~s}{ }^{3} \mathrm{~S}$ state. Low pressure flames provide stable sources of large concentrations of hot atoms. Using flame conditions identical to those used previously to study OH (Kohse-Höinghaus et al, 1989), HCO (Jeffries et al, 1990), and NO (Heard et al, 1992), we have gas temperature measurements and model predictions of the gas composition from this previous work..

Simultaneous $\mathrm{H}$ atom LIF and ASE were observed in all the flames studied. Using our model predictions of the major species concentrations, we corrected the LIF signals for collisional quenching using the data of Meier et al. (1986). We found a serious discrepancy between our model prediction and the measured $\mathrm{H}$ atom concentration (Jeffries et al, 1990). We discovered that our low-pressure premixed flames were not well described by a one-dimensional fluid model and that radial transport must be included. Radial transport increases the diameter of the flame and reduces the average flow velocity, which increases the reaction time and produces a flame closer to the burner surface than predicted by a one-dimensional model. Corrections to our fluid flow model have significantly improved the agreement between measurements and prediction for all the radical intermediate species previously measured in our laboratory for flames below 10 Torr.

The Balmer $\alpha$ ASE from two-photon excited atomic hydrogen is diverged through an etalon and the interference fringes are imaged onto a two-dimensional CCD array camera. We have made careful measurements of the bandwidth of the ASE signal excited with a laser power near threshold in $25 \mathrm{Torr}, 1200 \mathrm{~K} \mathrm{H} / \mathrm{O}_{2}$ flames and $1800 \mathrm{~K} \mathrm{CH}_{4} / \mathrm{O}_{2}$ flames. The velocity distribution 
of the atomic hydrogen in the two flames is significantly different. However, we find identical ASE bandwidth in both flames of about twice the expected Doppler width in the $1800 \mathrm{~K}$ flame. Thus, for atomic hydrogen, the ASE is broadened by mechanisms other than Doppler broadening.

Atomic oxygen appears to be a better candidate for gas temperature measurements from the ASE bandwidth. The difference in mass between $\mathrm{H}$ and $\mathrm{O}$ reduces the Doppler widths. For oxygen, we can excite the entire ground state velocity distribution or just a narrow slice of the velocity distribution by varying the bandwidth of the excitation laser light. We excite only one of the lower-state fine structure components. We have observed simultaneous ASE and LIF in our low-pressure $\mathrm{H}_{2} \mathrm{O}_{2}$ flame from atomic oxygen, and bandwidth measurements are under way.

We have modelled direct gain measurements on a seed laser beam from two-photon atom excitation. Here a cw probe laser is tuned to the wavelength of the atomic fluorescence and copropagated along the two-photon excitation laser. When the pulsed excitation laser produces excited states, the light from the $\mathrm{cw}$ laser will be amplified. The gain on the $\mathrm{cw}$ seed laser is a direct measure of the population inversion. The two-photon excitation cross section for atomic oxygen is known (Bamford et al., 1987) thus, the gain measurement is a direct measure of atom concentration. To understand how much gain to expect, we have developed a time-dependent computer model of the ASE signal from atomic oxygen. The model includes loss from the excited state collisional quenching, energy transfer from the triplet to quintet, photo-ionization, fluorescence, and ASE. We consider the linear excitation regime to eliminate Rabi flopping and other complicating nonlinear processes. For the oxygen concentration that we expect in our lowpressure flame, we predict that a 4-mJ, 10-ns excitation pulse pump will stimulate a $5 \%$ power gain in an $10-\mathrm{mW} \mathrm{cw}$ single-mode diode laser at $845 \mathrm{~nm}$.. The temporal profile of the gain follows that of the excitation pulse with less than 1-ns delay. The diode laser requirements are thus quite modest, and procurement of such a laser has begun.

\section{Conclusions}

\section{TASK 1:}

To date we have implemented a four-wave-mixing process to produce high powers in the vuv and used the resulting radiation to perform both $1+1 \mathrm{MPI}$ and two-photon-excited fluorescence in the vuv. Excitation of atomic ions will require higher powers than we have now. Previous work (Faris et al., 1991) indicates that higher power may be produced with our lasers with improvement in phase-matching. Our goals are to use this approach to improve the vuv power and to apply this radiation to two-photon excitation of atomic ions. 


\section{TASK 2:}

We have demonstrated that simultaneous ASE and LIF measurements are feasible from a variety of low-pressure flames. These environments have previously been well characterized and can now provide a testing ground for quantitative ASE measurements. Single-laser-pulse measurements of the ASE bandwidth have been demonstrated and may provide a method for single color gas temperature measurements. A time-dependent computer model for ASE has been assembled, and calculations show that direct gain measurements are possible for modest laser powers. 


\section{PERSONNEL}

The following professional scientists participated in the research supported by this contract:

Gregory W. Faris, Physicist, Co-Principal Investigator; Task Leader and lead experimentalist for Task 1.

Mark J. Dyer, Physics Associate Specialist in lasers and nonlinear optics; made many of the major technical accomplishments in Task 1.

David L. Huestis, Associate Director of the Molecular Physics Laboratory, Co-Principal Investigator, project supervisor, and Task 1 technical contributor, especially on theory and spectroscopy.

Jay B. Jeffries, Senior Chemical Physicist, Co-Principal Investigator; Task Leader for Task 2.

Dwayne E. Heard, Postdoctoral Fellow Laboratory Scientist for Task 2.

Michael S. Brown, Postdoctoral Fellow, Laboratory Scientist for Task 2; modeled probe laser gain in atomic oxygen with ASE.

Gregory P. Smith, Senior Chemical Physicist for Task 2. Performed chemical model calculations to obtain the partial pressures of flame species. 


\section{PUBLICATIONS}

The following publications were published or submitted for publication on research supported by this contract:

1. Gregory W. Faris and Mark J. Dyer, "Multiphoton Spectroscopy Using Tunable VUV Radiation from a Raman-Shifted Excimer Laser," in Short Wavelength Coherent Radiation: Generation and Applications, Philip H. Buckbaum and Natale M. Ceglio, Eds. (Optical Society of America, Washington, DC, 1991).

2. Gregory W. Faris, Mark J. Dyer, David L. Huestis, and William K. Bischel, "Two-Photon Spectroscopy of the $F^{1} \Pi_{g}$ and $f^{3} \Pi_{g}$ States of Molecular Fluorine," submitted to the Journal of Chemical Physics, 1992.

3. G. W. Faris and P. C. Cosby, "Observation of NO $B^{2} \Pi(v=3) \leftarrow X^{2} \Pi(v=0)$ Absorptions with 1+1 Multiphoton Ionization: Precision Line Position Measurements and Parity Assignment of the B2 $\Pi$ State," submitted to the Journal of Chemical Physics, 1992.

4. J. B. Jeffries, G. P. Smith, D. E. Heard, and D. R. Crosley, "Comparing Laser-Induced Fluorescence Measurements and Computer Models of Low Pressure Flame Chemistry," Ber. Bunsenges. Phys. Chem., submitted, 1992. 


\section{PRESENTATIONS}

The following conference papers were presented on research supported by this contract.

1. Gregory W. Faris and Mark J. Dyer, "Multiphoton Spectroscopy at $147 \mathrm{~nm}$ Using TwoPhoton-Resonant Difference Frequency Mixing," Paper QTuI30, Quantum Electronics and Laser Science Conference, Anaheim, CA, May 12-14, 1992.

2. D. Huestis, G. Faris, and J. Jeffries, "Novel Nonlinear Laser Diagnostic Techniques," AFOSR Contractors Meeting in Propulsion, La Jolla, CA, June 15-19, 1992.

3. D. E. Heard and J. B. Jeffries, "Laser Excited Amplified Spontaneous Emission of Atomic Hydrogen in Low-Pressure Flames," Gordon Conference on the Physics and Chemistry of Laser Diagnostics in Combustion, Plymouth, NH, July 1991.

4. D. E. Heard and J. B. Jeffries, "Amplified Spontaneous Emission Measurements of Atomic Hydrogen in Low-Pressure Flames," VII Interdisciplinary Laser Science Conference, Bull. Amer. Phy. Soc. 361950 (1991).

5. D. E. Heard and J. B. Jeffries, "Laser-Excited Amplified Spontaneous Emission of Atomic Hydrogen in Low-Pressure Flames," Annual Meeting of the Optical Society of America, San Jose, CA, November 1991.

6. D. E. Heard and J. B. Jeffries, "Laser Excited Amplified Spontaneous Emission of Atoms in Low-Pressure Flames," Optical Society of America, CLEO, May 1992.

7. M. S. Brown and J. B. Jeffries, "Investigation of Stimulated Emission as an Optical Diagnostic of Reacting Flows," International Laser Science Conference '92, Society of America, Albuquerque, NM, September 1992.

8. J. B. Jeffries, D. E. Heard, and M. S. Brown, "Amplified Spontaneous Emission Measurements of Atomic Oxygen and Hydrogen," 31st AIAA Aerospace Sciences Meeting, Reno, Nevada, January 1993. 


\section{PROJECT INTERACTIONS}

We have served as informal advisors on aspects of the work supported by this contract in the following technical interactions.

Professor Terry Cool of Cornell University; visit to SRI International on November 4, 1991, discussed generation of high power vuv for trace analysis.

Professor Edward Eyler of the Department of Physics and Astronomy, the University of Delaware, telephone conversation on high power vuv for basic physics measurements.

Masayuki Katehara, Keio University, Japan, visit to SRI International, May 19, 1992, discussions on spectroscopy of molecular fluorine.

Michael Casassa, NIST, Gaithersburg, telephone conversation on June 1, 1992 on $F_{2}$ operation using excimer laser systems.

In May, 1992 Conference on Lasers and Electro-Optics and Quantum Electronic and Laser Science Conference, in Anaheim, California conversations were held with:

1. Masayuki Katehara, Keio University, Japan, on vuv lasers.

2. Professor Edward Eyler of the University of Delaware on vuv generation and techniques.

3. Bruce Hudson of the University of Oregon on high power vuv for electronic Raman scattering.

In June, 1992 AFOSR Contractors Meeting in Propulsion, at the , La Jolla, California, conversations were held with:

1. Dr. Bish Ganguly of Wright-Patterson on AFB ion detection and interactions.

2. Professor Robert Pitz of Vanderbilt University on nonlinear optical diagnostic techniques.

3. Dr. H. F. Calcote of Aerochem Research Laboratories on ion interactions and soot formation.

4. Professor John Daily of the University of Colorado on coherent transient diagnostic techniques. 
The principles of amplified spontaneous emission were discussed with numerous visitors to SRI during the past year and with many colleagues at several conferences. The most notable interactions are summarized below.

At the Cordon Conference on the Physics and Chemistry of Laser Diagnostics for Combustion in July, 1991 a lengthy discussion was conducted on the problems for quantitative ASE with Dr. Marcus Alden and Dr. Ulf Westblom from Lund Institute of Technology, Dr. John Goldsmith of Sandia National Laboratory, and Dr. Katharina Kohse-Hoinghaus of DLR. All these scientists have on-going research on various aspects of ASE.

In September 1991, at the International Laser Science Conference, ASE was again the subject of lengthy discussions with Dr. Ingrid Wysong trom Phillips Lab, Dr. Andy Sappey from Los Alamos, Dr. Mark Crofton from Aerospace, and Dr. Robert Lucht from Sandia National Laboratory.

In November 1991, at the Annual Meeting of the Optical Society of America, ASE was discussed with Dr. Terry Cool of Cornell, Dr. Rosario Sausa and Dr. Andrzej Miziolek of the U.S. Army Ballistics Research Laboratory.

In January 1992 at the OSA topical meeting on laser applications to chemical analysis, ASE was again discussed with Dr. Alan Eckbreth of UTRC, Dr. Westblom of Lund, and Dr. Miziolek of BRL.

In May 1992 at CLEO, new results with ASE on atomic oxygen were discussed at length with Dr. Rober Lucht and Dr. John Goldsmith of Sandia National Laboratory. 


\section{INVENTIONS}

No inventions were disclosed under this contract during the past year. 


\section{REFERENCES}

Bamford, D. J. M. J. Dyer, and W. K. Bischel, "Single Frequency Laser Measurements of TwoPhoton Cross Sections and Doppler-Free Spectra for Atomic Oxygen," Phys. Rev. A 36, 3497-3500 (1987).

Faris, G. W. and Mark J. Dyer, "Multiphoton Spectroscopy Using Tunable VUV Radiation from a Raman-Shifted Excimer Laser," in Short Wavelength Coherent Radiarion: Generation and Applications, Philip H. Buckbaum and Natale M. Ceglio, Eds. (Optical Society of America, Washington, DC, 1991).

G. W. Faris, M. J. Dyer, W. K. Bischel, and D. L. Huestis, "Multiphoton Detection Techniques for $F$ and $F_{2}$," Final Report, AFOSR Contract No. F49620-88-K-0003, SRI International, Menlo Park, CA (November 1990).

G. W. Faris, J. B. Jeffries, and D. L. Huestis, Annual Technical Report, AFOSR Contract No. F49620-90-C-0044, SRI International, Menlo Park, CA (June 1991).

D. E. Heard, J. B. Jeffries, G. P. Smith, and D. R. Crosley, "LIF Measurements in Methae/Air Flames of Radicals Important in Prompt-NO Formation," Combustion and Flame 88, 137148 (1992).

G. Hilber, A. Lago, and R. Wallenstein, "Broadly Tunable Vacuum-Ultraviolet/ExuremeUltraviolet Radiation Generated by Resonant Third-Order Frequency Conversion in Krypton," J. Opt. Soc. Am. B, 4 , 1753-1764 (1987).

R. Hilbig, G. Hilber, A. Lago, B. Wolff, and R. Wallenstein, "Tunable Coherent VUV Radiation Generated by Nonlinear Optical Frequency Conversion in Gases," Comments At. Mol. Phys. 18, 157-180 (1986).

J. B. Jeffries, D. R. Crosley, I. J. Wysong, and G. P. Smith, "Laser-Induced Fluorescence Detection of HCO in a Low-Pressure Flame," 23d Symposium (International) on Combustion, The Combustion Institute, Pittsburgh, PA, 1990, P. 1847-1854.

K. Kohse-Höinghaus, J. B. Jeffries, R. A. Copeland, G. P. Smith, and D. R. Crosley, "The Quantitative LIF Determination of OH Concentrations in Low-Pressure Flames," 22d Symposium (International) on Combustion, The Combustion Institute, Pittsburgh, PA, 1989, P. 1857-1866.

J. P. Marangos, N. Shen, H. Ma, M. H. R. Hutchinson, and J. P. Connerade, "Broadly Tunable Vacuum-Ultraviolet Radiation Source Employing Resonant Enhanced Sum-Difference Frequency Mixing in Krypton," J. Opt. Soc. Am. B 7, 1254-1259 (1990).

U. Meier, K. Kohse-Höhaus, and Th. Just, "Hand O Atom Detection for Combustion Applications: Study of Quenching and Laser Photolysis Effects" Chem. Phys. Lett. 126, 567 (1986). 
T. Okada, Y Hirakawa, and M. Maeda, "Generation of Tunable XUV Radiation by Two-Photon Resonant Four-Wave Mixing in $\mathrm{H}_{2}$, "Paper CThD6 presented at the Conference on Lasers and Electro-Optics 1990, Anaheim, California, May 21-25, 1990.

H. Pummer, H. Egger, T. S. Luk, T. Srinivasan, and C. K. Rhodes, "Vacuum-Ultraviolet Stimulated Emission from Two-Photon-Excited Molecular Hydrogen," Phys. Rev. A 28, 795-801 (1983).

C.E.M. Strauss and D. J. Funk,"Bradly Tunable Difference-Frequency Generation of VUV Using Two-Photon Resonances in $\mathrm{H}_{2}$ and Kr," Opt. Lett. 16, 1192 (1991). 